\title{
AN EMBEDDING SPACE TRIPLE OF THE UNIT INTERVAL INTO A GRAPH AND ITS BUNDLE STRUCTURE
}

\author{
KATSURO SAKAI
}

(Communicated by James E. West)

Abstract. Let $l_{2}$ denote a Hilbert space, and let

$$
\begin{aligned}
& l_{2}^{Q}=\left\{\left(x_{i}\right) \in l_{2}|\sup | i \cdot x_{i} \mid<\infty\right\} \text { and } \\
& l_{2}^{f}=\left\{\left(x_{i}\right) \in l_{2} \mid x_{i}=0 \text { except for finitely many } i\right\} .
\end{aligned}
$$

We show that the triple $\left(H(X), H^{\mathrm{LIP}}(X), H^{\mathrm{PL}}(X)\right)$ of spaces of homeomorphisms, of Lipschitz homeomorphisms, and of PL homeomorphisms of a finite graph $X$ onto itself is an $\left(l_{2}, l_{2}^{Q}, l_{2}^{f}\right)$-manifold triple, and that the triple $\left(E(I, X), E^{\mathrm{LIP}}(I, X), E^{\mathrm{PL}}(I, X)\right)$ of spaces of embeddings, of Lipschitz embeddings, and of PL embeddings of $I=[0,1]$ into a graph $X$ is an $\left(l_{2}, l_{2}^{Q}, l_{2}^{4}\right)$ manifold triple.

Let $l_{2}$ denote a Hilbert space, and let

$$
l_{2}^{Q}=\left\{\left(x_{i}\right) \in l_{2}|\sup | i \cdot x_{i} \mid<\infty\right\}
$$

and

$$
l_{2}^{f}=\left\{\left(x_{i}\right) \in l_{2} \mid x_{i}=0 \text { except for finitely many } i\right\} .
$$

An $\left(l_{2}, l_{2}^{Q}, l_{2}^{f}\right)$-manifold triple is defined as a triple $(M, N, W)$ of an $l_{2}$ manifold, an $l_{2}^{Q}$-manifold, and an $l_{2}^{f}$-manifold which admits an open cover $\mathscr{U}$ of $M$ and open embeddings $\varphi_{U}: U \rightarrow l_{2}, U \in \mathscr{U}$, such that $\varphi_{U}(U \cap N)=$ $\varphi_{U}(U) \cap l_{2}^{Q}$ and $\varphi_{U}(U \cap W)=\varphi_{U}(U) \cap l_{2}^{f}\left[\mathrm{SW}_{2}\right]$. If $X$ is a compact Euclidean polyhedron with $\operatorname{dim} X>0$ and $Y$ is an open set in $\mathbb{R}^{n}$, the triple $(C(X, Y), \operatorname{LIP}(X, Y), \operatorname{PL}(X, Y))$ of spaces of (continuous maps), of Lipschitz maps, and of PL maps of $X$ into $Y$ is such a manifold triple [Sa]. In this note, we find other examples of such manifold triples of function spaces where every function space has the compact-open topology; that is, we have the

Received by the editors July 27, 1989 and, in revised form, February 18, 1990; presented to the Mathematical Society of Japan, April 2, 1990.

1980 Mathematics Subject Classification (1985 Revision). Primary 58D05, 58D10, 57N20, 54F50; Secondary 54B20.

Key words and phrases. $\left(l_{2}, l_{2}^{Q}, l_{2}^{f}\right)$-manifold triple, graph, space of homeomorphisms, Lipschitz homeomorphism, PL homeomorphism, space of embeddings, Lipschitz embedding, PL embedding, space of arcs, local trivial bundle. 
following:

Theorem 1. For a finite graph (polyhedron of $\operatorname{dim}=1$ ) $X \subset \mathbb{R}^{n}$, the triple $\left(H(X), H^{\mathrm{LIP}}(X), H^{\mathrm{PL}}(X)\right)$ of the spaces of homeomorphisms, of Lipschitz homeomorphisms and of PL homeomorphisms of $X$ onto itself is an $\left(l_{2}, l_{2}^{Q}, l_{2}^{f}\right)$ manifold triple.

Theorem 2. For a graph $X \subset \mathbb{R}^{n}$, the triple $\left(E(I, X), E^{\mathrm{LIP}}(I, X), E^{\mathrm{PL}}(I, X)\right)$ of spaces of embeddings, of Lipschitz embeddings, and of PL embeddings of $I=[0,1]$ into $X$ is an $\left(l_{2}, l_{2}^{Q}, l_{2}^{f}\right)$-manifold triple.

By Theorem 1, Conjecture 2.6 in $\left[\mathrm{SW}_{1}\right]$ is true in the 1-dim case. Let $H_{\partial}(I)=\{h \in H(I)|h| \partial I=\mathrm{id}\}$, where $\partial I=\{0,1\}$. Similarly, $H_{\partial}^{\mathrm{LIP}}(I)$ and $H_{\partial}^{\mathrm{PL}}(I)$ are defined. First we prove the following:

Theorem 3. $\left(H_{\partial}(I), H_{\partial}^{\mathrm{LIP}}(I), H_{\partial}^{\mathrm{PL}}(I)\right)$ is homeomorphic $(\cong)$ to $\left(l_{2}, l_{2}^{Q}, l_{2}^{f}\right)$. Proof. For simplicity, let $H=H_{\partial}(I), H^{\prime}=H_{\partial}^{\mathrm{LIP}}(I), H^{\prime \prime}=H_{\partial}^{\mathrm{PL}}(I)$. For each $m \in \mathbb{N}$, let $L_{m}=\left\{h \in H^{\prime} \mid\right.$ bilip $\left.h \leq 1+m\right\}$, where bilip $h$ is the minimum of $k \geq 1$ such that

$$
k^{-1} \cdot|x-y| \leq|h(x)-h(y)| \leq k \cdot|x-y| \quad \text { for each } x, y \in I .
$$

If $m<m^{\prime}$, then $L_{m}$ is a $Z$-set in $L_{m^{\prime}}$. In fact, we have a homotopy $\varphi: L_{m^{\prime}} \times$ $I \rightarrow L_{m^{\prime}}$ defined as follows:

$$
\begin{aligned}
\varphi_{t}(h)(s)= & \left(1-\frac{t}{2}\right) \cdot h\left(\frac{s}{1-t / 2}\right) \quad \text { for } 0 \leq s \leq 1-\frac{t}{2}, \\
& \varphi_{t}(h)\left(1-\frac{t}{4}\right)=1-\frac{\left(1+m^{\prime}\right) \cdot t}{4},
\end{aligned}
$$

and

$$
\varphi_{t}(h) \text { is linear on }\left[1-\frac{t}{2}, 1-\frac{t}{4}\right] \text { and on }\left[1-\frac{t}{4}, 1\right] \text {, }
$$

which satisfies $\varphi_{0}=\mathrm{id}$ and $\operatorname{Im}\left(\varphi_{t}\right) \cap L_{m}=\varnothing$ if $t>0$. Since $H^{\prime}=\bigcup_{m \in \mathbb{N}} L_{m}$ and each $L_{m}$ is a compact convex set in the Banach space $C(I, \mathbb{R})$, which contains an infinite-dimensional, $\sigma$-fd-compact, convex set $H^{\prime \prime} \cap L_{m}$ as a dense subset, $\left(L_{m}, H^{\prime \prime} \cap L_{m}\right) \cong(Q, \sigma)$ by [Do, Theorem 2(i)]. Thus the tower $\left\{L_{m}\right\}_{m \in \mathbb{N}}$ satisfies the condition $(* *)$ in $\left[\mathrm{SW}_{2}\right]$. Let $\psi_{n}: H \rightarrow H^{\prime \prime}, n \in \mathbb{N}$, be maps such that $\psi_{n}(h)\left(\frac{i}{n}\right)=h\left(\frac{i}{n}\right)$ and $\psi_{n}(h)$ is linear on each $\left[\frac{i-1}{n}, \frac{i}{n}\right]$. Then $\psi_{n}$ converges to id as $n \rightarrow \infty$. For each $h \in H$, bilip $\psi_{n}(h)=\max \left\{a, b^{-1}\right\}$, where

$$
\begin{aligned}
& a=\max \left\{n \cdot\left(\psi_{n}(h)\left(\frac{i}{n}\right)-\psi_{n}(h)\left(\frac{i-1}{n}\right)\right) \mid i=1, \ldots, n\right\}, \\
& b=\min \left\{n \cdot\left(\psi_{n}(h)\left(\frac{i}{n}\right)-\psi_{n}(h)\left(\frac{i-1}{n}\right)\right) \mid i=1, \ldots, n\right\} .
\end{aligned}
$$

Hence bilip $\psi_{n}(h) \leq$ bilip $h$. It follows that $H^{\prime}$ is map dense in $H$ and $\left\{L_{m}\right\}_{m \in \mathbb{N}}$ satisfies the condition $(*)^{\prime}$ in $\left[\mathrm{SW}_{2}\right]$. Since $H \cong l_{2}$ by [An] [cf. 
$\mathrm{Ke}],\left(H, H^{\prime}, H^{\prime \prime}\right) \cong\left(l_{2}, l_{2}^{Q}, l_{2}^{f}\right)$ by $\left[\mathrm{SW}_{2}\right.$, Lemma 1.5 and Theorems 2.1 and 2.2].

By the arguments in [An], Theorem 1 follows from Theorem 3. Since [An] is unpublished, we give a sketch of the proof for the reader: Let $A, B$, and $C$ be the sets of isolated points, of end points, and of branch points of $X$, respectively. Let $D$ be the set of components of $X$ that are simple closed curve. Let $E$ and $F$ be the sets of maximal free open arcs in $X \backslash \cup D$ such that the closure of each member of $E$ is an arc and the closure of each member of $F$ a simple closed curve. Let $W$ be the finite set of all permutations of the union $A \cup B \cup C \cup D \cup E \cup F$ onto itself which carries each $A, B, C, D, E$, and $F$ onto itself and preserves incidence in $X$, and let $n(W), n(D), n(E)$, and $n(F)$ denote the numbers of elements in $W, D, E$, and $F$, respectively. Let $T$ be a finite space of $n(W) \cdot 2^{n(D)+n(F)}$ points, and let $V$ be the product space of $n(D)$ circles except where $n(D)=0$, in which case it is a single point. Then it is not hard to see that

$$
\left(H(X), H^{\mathrm{LIP}}(X), H^{\mathrm{PL}}(X)\right) \cong\left(H \times T \times V, H^{\prime} \times T \times V, H^{\prime \prime} \times T \times V\right),
$$

which implies Theorem 1 by Theorem 3.

We prove Theorem 2 in a more general setting. To this end, we extend the piecewise linearity to maps from $I$ to a metric space $X=(X, d)$. A map $f:[a, b] \rightarrow X$ is said to be linear if

$$
\frac{d(f(t), f(a))}{d(f(t), f(b))}=\frac{|t-a|}{|t-b|} \quad \text { for each } a<t<b,
$$

and $f:[a, b] \rightarrow X$ is piecewise linear (PL) if there is a sequence $a=s_{0}<s_{1}<$ $\cdots<s_{n}=b$ such that each $f \mid\left[s_{i-1}, s_{i}\right]$ is linear. The space of PL embeddings of $I$ into a metric space $X$ is also denoted by $E^{\mathrm{PL}}(I, X)$. In case $X$ is a connected polyhedron in $\mathbb{R}^{n}$, we adopt the arc-length metric $d$ defined by using Euclidean metric. Then the piecewise linearity of $f: I \rightarrow X$ defined above coincides with the usual sense. In this case, $E^{\mathrm{LIP}}(I, X)$ is not changed, since $d$ is locally Lipschitz-equivalent to the Euclidean metric by [LV, Theorem 2.34]. Note that this metric $d$ is convex; that is, for each $x, y \in X$ there is some $z \in X$ such that $d(x, z)=d(y, z)=d(x, y) / 2$. Let $a(X)$ denote the hyperspace of arcs in $X$ with the Vietoris topology (Hausdorff metric) and $\operatorname{Im}: E(I, X) \rightarrow a(X)$ the natural map defined by $\operatorname{Im}(h)=h(I)$. Theorem 2 is a corollary to the following:

Theorem 4. Let $X$ be a locally compact $1-\operatorname{dim} \operatorname{ANR} X$ with a metric $d$ which is convex on a neighborhood of each point. Then $\left(E(I, X), E^{\mathrm{LIP}}(I, X)\right.$, $\left.E^{\mathrm{PL}}(X, Y)\right)$ is an $\left(l_{2}, l_{2}^{Q}, l_{2}^{f}\right)$-manifold triple and the map $\operatorname{Im}: E(I, X) \rightarrow$ $a(X)$ is a locally trivial bundle with fiber $\mathbb{Z}_{2} \times l_{2}$ and $\operatorname{Im} \mid E^{\mathrm{LIP}}(I, X)$ and $\operatorname{Im} \mid E^{\mathrm{PL}}(I, X)$ are subbundles with fibers $\mathbb{Z}_{2} \times l_{2}^{Q}$ and $\mathbb{Z}_{2} \times l_{2}^{f}$, respectively. 
Before the proof, note that any connected locally compact 1-dim ANR has a convex metric. In fact, it has a Peano compactification with locally nonseparating remainder by $[\mathrm{Cu}]$ and any Peano continuum admits a convex metric by [Bi] or [Mo].

In Theorem 4, for each $h \in E(I, X)$, there is a dendrite (=compact 1$\operatorname{dim} \mathrm{AR}) \quad Y$ such that $h(I) \subset$ int $Y$, whence $E(I, Y)$ is a neighborhood of $h$ in $E(I, X)$. The arc-length metric of $Y$ defined by using the metric of $X$ is convex and locally coincides with the metric of $X$. Thus Theorem 4 reduces to the case in which $X$ is a dendrite with a convex metric.

Theorem 5. For a dendrite $X$ with a convex metric, $\left(E(I, X), E^{\mathrm{LIP}}(I, X)\right.$, $\left.E^{\mathrm{PL}}(I, X)\right)$ is an $\left(l_{2}, l_{2}^{Q}, l_{2}^{f}\right)$-manifold triple and the map $\mathrm{Im}: E(I, X) \rightarrow$ $a(X)$ is a locally trivial bundle with fiber $\mathbb{Z}_{2} \times l_{2}$ and $\operatorname{Im} \mid E^{\mathrm{LIP}}(I, X)$ and $\operatorname{Im} \mid E^{\mathrm{PL}}(I, X)$ are subbundles with fibers $\mathbb{Z}_{2} \times l_{2}^{Q}$ and $\mathbb{Z}_{2} \times l_{2}^{f}$, respectively.

Proof. For simplicity, let $E=E(I, X), E^{\prime}=E^{\mathrm{LIP}}(I, X)$ and $E^{\prime \prime}=E^{\mathrm{PL}}(I, X)$ and let $H, H^{\prime}$ and $H^{\prime \prime}$ be as in the proof of Theorem 1.1. Let $b(X)=X^{2} \backslash \Delta X$, where $\Delta X$ is the diagonal of $X^{2}$, and let $\beta: E \rightarrow b(X)$ be the map defined by $\beta(h)=(h(0), h(1))$. We have the map $\alpha: b(X) \rightarrow a(X)$ such that $\alpha(x, y)$ is the unique arc in $X$ connecting $x$ and $y$. Then $\alpha \circ \beta=\operatorname{Im}: E(I, X) \rightarrow a(X)$, and $\alpha$ is a locally trivial bundle with fiber $\mathbb{Z}_{2}$. (Geometrically, $b(X)$ can be considered as the space of oriented arcs in $X$.) Hence it suffices to construct a homeomorphism

$$
\varphi:\left(E, E^{\prime}, E^{\prime \prime}\right) \rightarrow\left(b(X) \times H, b(X) \times H^{\prime}, b(X) \times H^{\prime \prime}\right)
$$

so that $p \circ \varphi=\beta$, where $p: b(X) \times H \rightarrow b(X)$ is the projection. Then $\left(E, E^{\prime}, E^{\prime \prime}\right)$ is an $\left(l_{2}, l_{2}^{Q}, l_{2}^{f}\right)$-manifold triple by Theorem 3 and the result of $\left[\mathrm{SW}_{2}\right]$. From the uniquely arcwise connectedness of $X$, there exists a map $\lambda: X^{2} \times I \rightarrow X$ such that

$$
d(x, \lambda(x, y, t))=t \cdot d(x, y) \quad \text { and } \quad d(y, \lambda(x, y, t))=(1-t) \cdot d(x, y)
$$

for each $x, y \in X$ and $t \in I$. We define the map $\tau: b(X) \rightarrow E$ by $\tau(x, y)(t)=$ $\lambda(x, y, t)$. As is easily observed, $\tau(b(X)) \subset E^{\prime \prime}$. From the uniquely arcwise connectedness of $X, \tau \circ \beta(h)(I)=h(I)$ for each $h \in E$. Then the desired homeomorphism $\varphi$ and its inverse are defined by

$$
\varphi(h)=\left(\beta(h),(\tau \circ \beta(h))^{-1} \circ h\right) \text { and } \varphi^{-1}(x, y, g)=\tau(x, y) \circ g .
$$

Example. If $X$ contains a two-disk, both Im: $E(I, X) \rightarrow a(X)$ and Im: $E^{\mathrm{LIP}}(I, X) \rightarrow a(X)$ are not locally trivial bundles. To show this, let $g: I^{2} \rightarrow X$ be an embedding and $A_{0}=g(I \times\{0\})$. For each $n \in \mathbb{N}$, let

$$
A_{n}=g\left(I \times\left\{\frac{1}{2 n}, \frac{1}{2 n-1}\right\} \cup\{1\} \times\left[\frac{1}{2 n}, \frac{1}{2 n-1}\right]\right) \in a(X) .
$$

Then $A_{n}$ converges to $A_{0}$ in $a(X)$. However, any $h_{n} \in \operatorname{Im}^{-1}\left(A_{n}\right) \quad(n \in \mathbb{N})$ does not converge to any $h \in \operatorname{Im}^{-1}\left(A_{0}\right)$, because $\left\{h_{n}(0), h_{n}(1)\right\}$ converges to 
$\{g(0,0)\}$, but $\{h(0), h(1)\}=\{g(0,0), g(1,0)\}$. In case $X$ is a polyhedron with $\operatorname{dim} X>1, \operatorname{Im}: E^{\mathrm{PL}}(I, X) \rightarrow a^{\mathrm{Pol}}(X)$ is not a locally trivial bundle, where $a^{\mathrm{Pol}}(X)$ is the subspace of $a(X)$ consisting of polyhedral arcs.

Problem. Let $X$ and $Y$ be Euclidean polyhedra such that $X$ is compact and $E(X, Y) \neq \varnothing$. Is $\left(E(X, Y), E^{\mathrm{LIP}}(X, Y), E^{\mathrm{PL}}(X, Y)\right)$ an $\left(l_{2}, l_{2}^{Q}, l_{2}^{f}\right)$-manifold triple? Is each space of this triple an ANR? If $X=I$ and $\operatorname{dim} Y>1$, is it then an ANR?

Remark. In Theorem 5, Im: $E(I, X) \rightarrow a(X)$ is nontrivial in the case $X \not ⿻ I$. In fact, $\operatorname{Im}=\alpha \circ \beta$ and $\beta$ is trivial as shown in the proof, but $\alpha$ is nontrivial since $b(X)=X^{2} \backslash \Delta X$ is connected in this case. It should be noted that $a(X)$ is not contractible in general. For example, in the case $X$ is the simple triod, $a(X)$ has the homotopy type of $S^{1}$.

The author would like to express his thanks to R. Cauty for his remark.

\section{REFERENCES}

[An] R. D. Anderson, Spaces of homeomorphisms of finite graphs, unpublished manuscript.

[Bi] R. H. Bing, Partitioning a set, Bull. Amer. Math. Soc. 55 (1949), 1101-1110.

[Cu] D. W. Curtis, Hyperspaces of noncompact metric spaces, Compositio Math. 44 (1980), 139152.

[Do] T. Dobrowolski, The compact Z-set property in convex sets, Topology and Appl. 23 (1986), 163-172.

[Ke] J. Keesling, Using flows to construct Hilbert space factors of function spaces, Trans. Amer. Math. Soc. 161 (1971), 1-24.

[LV] J. Luukkainen and J. Väisälä, Elements of Lipschitz topology, Ann. Acad. Sci. Fenn. Ser. A. I. Math. 3 (1977), 85-122.

[Mo] E. E. Moise, Grille decomposition and convexification theorems for compact locally connected continua, Bull. Amer. Math. Soc. 55 (1949), 1111-1121.

[Sa] K. Sakai, A function space triple of a compact polyhedron into an open set in Euclidean space, Proc. Amer. Math. Soc. 108 (1990), 547-555.

[SW $\left.\mathrm{SW}_{1}\right] \mathrm{K}$. Sakai and R. Y. Wong, On the space of Lipschitz homeomorphisms of a compact polyhedron, Pacific J. Math. 139 (1989), 195-207. $\left[\mathrm{SW}_{2}\right] \frac{}{555 .}$, On infinite-dimensional manifold triples, Trans. Amer. Math. Soc. 318 (1990), 545-

Institute of Mathematics, Uiviversity of Tsukuba, Tsukuba City, 305 Japan 